\title{
Risk factors and prevalence of porcine circovirus 2 and porcine reproductive and respiratory virus in 59 Greek pig farms
}

\author{
VASILEIOS PAPATSIROS ${ }^{1}$, Georgios Papakonstantinou ${ }^{2}$, Charalambos Billinis ${ }^{3}$, \\ Eletherios Meletis ${ }^{2}$, Georgios Maragkakis ${ }^{2}$, Nikolaos Tsekouras ${ }^{2}$, Dimitra Bitchava ${ }^{4}$, and \\ Polychronis Kostoulas ${ }^{2}$ \\ ${ }^{1}$ University of Thessaly School of Health Sciences \\ ${ }^{2}$ University of Thessaly \\ ${ }^{3}$ Faculty of Veterinary Medicine, University of Thessaly \\ ${ }^{4}$ Affiliation not available
}

November 16, 2020

\begin{abstract}
The objectives of the present study were to describe for first time the prevalence of porcine reproductive and respiratory syndrome virus (PRRSV) and porcine circovirus type-2 (PCV2) among pig farms in Greece and the association between risk factors and PRRSV, PCV2 PCR-status. PRRSV and PCV2 are the leading causes of huge financial losses in swine industry worldwide. Despite the implementation of control measures, both remain a major problem for the majority of pig farms. Identification of risk factors, which can lead to PRRSV and/or PCV2 infection, could be useful in preventing it. The study included 59 pig farms, across Greece, with a total population of 22.500 sows, which represent about $40 \%$ of the entire sow capacity of Greek swine production. Data regarding herd health management protocols were collected from each farm. Additionally, blood samples from breeding stock, weaners, growers and finishers were taken from each farm. The sera were tested for PRRSV and PCV2, the results indicated that both viruses remain a major challenge for the Greek swine industry. Finally, main risk factors involved in the infection process by these viruses were identified and could be used for future monitoring both diseases. In particular, vaccination programs such as the mass PRRSV vaccination with modified-live virus (MLV) in breeding stock during the last stages of gestation or with killed-virus (KV) during the middle of gestation are more likely to be associated with PRRSV seropositivity. Farms with low biosecurity level are associated with higher PRRSV circulation. It has, also, been revealed that breeding stock is more likely to be associated with PCV2 active circulation compared to weaners and growers. In conclusion, our results could be the basis of the development of surveillance protocols for a national monitoring system for PRRSV and PCV2, which could prevent future infection of Greek farms.
\end{abstract}

\section{INTRODUCTION}

Porcine Reproductive and Respiratory Syndrome virus (PRRSV) causes clinical diseases in sows, and/or their piglets. PRRSV outbreaks remain a global problem for the swine industry worldwide, causing significant economic losses (Nieuwenhuis, Duinhof, \& van Nes, 2012; Papatsiros 2012a). The frequency of PRRSV outbreaks and the severity of clinical signs in a herd are driven by virulence of the particular PRRSV strain, vaccination protocols and risk factors, such as viral or bacterial co-infections or gilt and semen introduction (Baysinger et al., 1997; Papatsiros, 2012a; Rosendal et al., 2016). Identification of risk factors is important to identify and implement adequate control measures and to design cost effective surveillance strategies (Papatsiros, 2012a). Previous studies have been carried out to investigate risk factors for PRRSV infection at herd level. Increased herd size, distance to the nearest farm, pig and herd density, housing conditions, vaccination with modified live (MLV) PRRSV vaccines, and introduction of semen, boars, and 
gilts were found to be associated with increased risk of PRRSV infection (Weigel, Firkins, \& Scherba, 2000; Mortensen et al., 2002; Evans, Medley, \& Green, 2008; Velasova, Alarcon, Williamson, \& Wieland, 2012). Vaccination against PRRSV is commonly applied (Pileri \& Mateu, 2016), but does not always sufficiently prevent PRRSV outbreaks (Alexopoulos, Kritas, Kyriakis, Tzika, \& Kyriakis, 2005; Papatsiros, 2012a, b; Piontkowski, Kroll, Orveillon, Kraft, \& Coll, 2016; Nan et al., 2017; Kristensen et al., 2018; Oh, Seo, Park, \& Chae, 2019; Bitsouni, Lycett, Opriessnig, \& Doeschl-Wilson, 2019) neither controls the elimination of the virus circulation at farm level (Cano, Dee, Murtaugh, \& Pijoan, 2007). Despite the implementation of various control measures, including vaccination, changes to management practices or breed genetics, PRRSV continues to be a major health problem for pig farms worldwide (Mateu \& Diaz, 2008).

Porcine circovirus type 2 (PCV2) is the primary causative agent of several syndromes known as porcine circovirus-associated disease (PCVAD). PCVAD is currently considered one of the most relevant infectious diseases in the global swine industry not only as health problem but also from a financial point of view (Gillespie, Opriessnig, Meng, Pelzer, \& Buechner-Maxwell, 2009). As PCVAD is present worldwide, studies aimed at identifying risk factors associated with PCV2 infection, as well as minimizing and monitoring the associated risk factors mainly through vaccination protocols (Gillespie et al., 2009). The PRRSV and PCV2 co-infection field cases are common and contribute to polymicrobial disease syndromes, such as porcine respiratory disease complex (PRDC) (Opriessnig, Gimenez-Lirola, \& Halbur, 2011; Sun et al., 2015). Under field conditions synergistic effects occur during PRRSV and PCV2 co-infection, as the clinical signs and lesions in the co-infected pigs are more severe than that of PCV2 or PRRSV singularly infected pigs (Harms et al., 2001; Drolet, Larochelle, Morin, Delisle, \& Magar, 2003; Fan et al., 2013), while PRRSV mutation rates may be increased (Ouyang, Zhang, Liu, \& Ren, 2019). PCV2 infection increases clinical signs not only induced by PRRSV, but also and by other porcine viruses (e.g. swine influenza, pseudorabies virus, porcine parvovirus), and meanwhile, secondary bacterial infection and decreased health/growth performance can also enhance in vivo environment for PCV2 infection (Ouyang et al., 2019). PRRSV infection is considered to be a major trigger factor for postweaning multisystemic wasting syndrome (PMWS) in PCV-2-infected pigs, (Rose et al., 2003). Many studies reported beneficial effects of commercial vaccines against PCV2, including improvement of performance parameters and decrease of PCV2 prevalence and viremia under field conditions (Opriessnig, Gerber, Xiao, Mogler, \& Halbur 2014a; Opriessnig et al., 2014b; Kristensen, Baadsgaard, \& Toft, 2011; Martelli et al., 2011; Martelli et al., 2016; Chae, 2012; Segalés, 2015; Dvorak, Yang, Haley, Sharma, \& Murtaugh, 2016; Afghah, Webb, Meng, \& Ramamoorthy, 2017; Tzika et al., 2015; Tassis et al., 2017; Figueras-Gourgues et al., 2019). Moreover, PCV2 vaccination induces efficient neutralizing antibody response and significantly reduces PCV2-associated lesions and viremia in co-infected pigs with PCV2 and PRRSV (Opriessnig et al., 2008).

Even if PRRSV diagnostic tools being available worldwide, PRRSV monitoring is mainly performed at the individual farm level and following different criteria among different regions and farms (Torrents et al., 2019). Therefore, the lack of regular and periodic PRRSV monitoring at farm level leads to limited epidemiological data on PRRSV surveillance at regional or national levels (Torrents et al., 2019). Generally, the published data for epidemiological prevalence of PRRSV and/or PCV2 infection under field conditions, including large number of commercial farms, are limited. The objectives of the present study were to describe for first time the prevalence of PRRSV and PCV2 among pig farms in Greece and the association between different risk factors and vaccinations protocols, respectively, and PRRS and PCV2 PCR-status of these herds at different production stages (breeding stock, weaners, growers, fatteners).

\section{MATERIALS AND METHODS}

\subsection{Ethics}

All procedures during this clinical study were performed according to the Code of Practice for the Conduct of Clinical trials for Veterinary Medical Products and the Guide for the Care and Use of Agricultural Animals in Research and Teaching, and the animals were maintained in accordance with National and European animal Welfare requirements (OECD 2000, EMEA 2001, EC 2003, FASS 2010). All animal procedures regarding animal care and use were approved by the Ethical Committee of the Faculty of Veterinary Medicine, School 
of Health Sciences, University of Thessaly (Approval number: 65/26-02-2019).

2.2 Study farms and data collection

\subsubsection{Farms}

The present cross-sectional study was conducted in Greece, including a total of 59 pig farms. Inclusion and exclusion criteria for selected farms were, a minimum capacity of 40 sows, as well as the operation type of exclusive farrow-to-finish farms. The selected 59 farms had totally a population around 22.500 sows, which represents about $40 \%$ of entire capacity of Greek swine production. Moreover, only farms that the owner or pig farm manager agreed to participate in the study voluntarily, were finally included.

\subsubsection{Records / sampling}

Between June 2013 and January 2020, the herds were examined and sampled at least once, evenly distributed over the whole study period. Data collection of each farm included:

(a) filling in a paper-based questionnaire on herd health management parameters in personal interviews with the farmers or pig farm managers, including the following records; location of the farm (Northern/Central/Western/Southern Greece), farm sow capacity ([?]100, 100-250, 250-500, >500 sows), presence of secondary respiratory infection (Absent/Low/Medium/High), maintenance of breeding nucleus or not, introduction of replacement gilts or not, semen introduction or not, biosecurity level (No/Low/Adequate/High) and detailed vaccination program of sow and/or weaners against PRRSV and PCV2 (mass vaccination until 3 days before farrowing, mass vaccination until 95 days of gestation, vaccination at $60^{\text {th }}$ day of gestation and $6^{\text {th }}$ day of lactation).

(b) blood sampling from breeding stock, weaners, growers and finishers by puncture of the vena jugularis externa. Blood samples were collected from 4 age groups: breeding stock (gilts, sows during early gestation and lactation), weaners at 50-60 days of age, growers at 100-110 days of age and fatteners at 150-160 days of age. Totally, 12 blood samples were taken randomly from each age group, according to Duinhof, Van Schaik, Van Esch, \& Wellenberg (2011).

\subsubsection{Laboratory tests}

Real time polymerase chain reaction (RT PCR) for porcine circovirus 2 (PCV2) and porcine reproductive and respiratory virus (PRRSV, type 1 and type 2) was performed in pools (each containing 4 samples) from collected blood samples and cycle threshold (CT) values from each pool were available. RT PCR detects accumulation of fluorescent signal. CT is defined as the number of cycles required for the fluorescent signal to cross the threshold. Therefore, CT levels are inversely proportional to the amount of nucleic acid in the sample. Zero CT values were interpreted as negative / undetermined results.

\subsection{Data analysis}

\subsubsection{Statistical Analysis}

CT values for PCV2 and PRRSV from each pool were considered continuous data with a large proportion of values clustered at zero, skewing the non-zero values to the right (positive skew) (Figure 1). In such cases, two-part models can be applied, where the first part includes probit or logistic regression to predict the probability of occurrence of a positive value and the second part introduces linear regression to predict the level of positive values (Xie, McHugo, Sengupta, Clark, \& Drake, 2004). An important aspect of two-part models compared to logistic regression models is that information is not discarded, since in the second case the question asked is "whether or not" it occurred and not "how much" something occurred (Skampardonis, Sotiraki, Kostoulas, \& Leontides, 2012).

In this study, a two-part model (Incerti, 2015) was employed for both diseases, predicting the probability of occurrence of a positive CT value, in each pool, using logistic regression, in the first part of the model, while positive $\mathrm{CT}$ values were the response variable in the second part of the model. 


\subsubsection{Outcome Variables}

For each disease two outcome variables were used (i) zero or positive CT value and (ii) the log-transformation of the positive CT values of RT PCR.

\subsubsection{Explanatory variables}

The explanatory variables that were examined in this study were: secondary respiratory infections; farm scale; region; pool origin; vaccination program for PCV2; vaccination program for PRRSV; vaccination program (for both diseases); nucleus breeding; F1 introduction; semen introduction; biosecurity level. All potential predictors were categorized as discrete data (Table 1).

2.3.1.3 Model Implementation

Two-part models refer to a mixture of distributions that take the general form:

$f(y)=1-\operatorname{Pr}(Y>0)$ if $y=0$

OR

$$
f(y)=\operatorname{Pr}(Y>0) x h(y) \text { if } y>0
$$

where y are the observed CT values for PCV2 and PRRS from each pool, $\operatorname{Pr}(\mathrm{Y}>0)$ the probability of occurrence of a positive $\mathrm{CT}$ value and $\mathrm{h}(\mathrm{y})$ a probability density defined when $\mathrm{y}>0$.

For a random variable $Y_{i}$, which represents the amount of a quantity with observed $y_{i}$, for each pool, let $R_{i}$, represent the occurrence variable where:

$$
R_{i}=0 \text { if } Y_{i}=0
$$

OR

$$
R_{i}=1 \text { if } Y_{i}>0
$$

The conditional probability of $\mathrm{R}_{\mathrm{i}}$ is defined as:

$$
\operatorname{Pr}\left(R_{i}=r_{i} \mid \theta_{i}\right)=1-p_{\mathrm{i}}\left(\theta_{1}\right) \text { if } r_{i}=0
$$

OR

$$
\operatorname{Pr}\left(R_{i}=r_{i} \mid \theta_{i}\right)=p_{\mathrm{i}}\left(\theta_{1}\right) \text { if } r_{i}=1
$$

where $\vartheta_{1}$ is a vector of fixed occurrence effects.

Therefore, the logistic regression part (first equation of the two-part model) for occurrence, is defined as:

$\operatorname{Logit}\left(p_{\mathrm{i}}\left(\theta_{1}\right)\right)=\mathrm{a}_{1} \mathrm{X}_{1 \mathrm{i}}+\mathrm{b}_{1}$

where $\mathrm{X}_{1 \mathrm{i}}$ is a vector of covariates for occurrence.

The second (nonzero) part of the model is defined as:

$S_{i}=\left[Y_{i} / R i=1\right]$ is set to be the "intensity" variable with probability density function (p.d.f.) $f\left(s_{i} \mid \vartheta_{2}\right)$ for $\mathrm{s}_{\mathrm{i}}>0$, where $\vartheta_{2}$ is a vector of fixed intensity effects. Therefore, the lognormal model (second equation of the two-part model) for intensity is defined as: 
$\log \left(\mathrm{S}_{\mathrm{i}} / \vartheta_{2}\right) \sim \mathrm{N}\left(\mathrm{a}_{2} \mathrm{X}_{2 \mathrm{i}}+\mathrm{b}_{2}, \sigma^{2}\right)(2)$

where $\mathrm{X}_{2 \mathrm{i}}$ is a vector of covariates for intensity.

All candidate variables were initially screened, one-by-one, to both equations, with a significance level of 0.25 . Variables with $\mathrm{P}<0.25$ were then offered to the two-part model which was, subsequently reduced by backwards elimination, until only significant $(\mathrm{P}<0.05)$ variables remained.

For the logistic regression part, the reported estimate and 95\% confidence intervals (CIs) for each predictor was the odds ratio (OR), while for the linear regression part was the coefficient $\mathrm{a}_{2}$ in the equation (2), given that all the other predictors included in the model are held constant.

\subsection{Goodness of fit}

The logistic regression part was assessed by the Hosmer and Lemesbow goodness of fit test. P-value for PCV2 was 0.9676 and 0.7492 for PRRSV (significance level 0.05) (Hosmer \& Lemesbow, 1980).

The linear regression part was assessed by the Pearson's Chi-squared test. The reported p-values were 0.3394 for PCV2 and 0.331 for PRRSV (significance level 0.05) (Pearson, 1900).

\subsection{Statistical Software}

The two-part model was implemented in the RStudio programming environment (Core Team R, 2019).

\section{RESULTS}

The results for the effect of predictors associated for both the occurrence (part I) and intensity (part II) model for PCV2 and PRRSV are summarized in Table 2.

\subsection{PCV2}

\subsubsection{Logistic regression part}

Farms located in Western Greece are less likely to be PCV2 seropositive (OR 0.25 (95\% CI 0.07; 0.71)) compared to farms located in other regions of Greece (Southern / Northern / Eastern Greece). Also, pools from breeding stock are more likely to be associated with positive CT values compared to pools from weaners [OR 0.21 (95\% CI 0.09; 0.46) and growers (OR 0.36 (95\% CI 0.17; 0.75)]. Vaccination of sows for PRRSV and weaners for PCV2 is associated with small probability of PCV2 seropositivity compared to other applied vaccination programs (OR 0.39 (95\% CI $0.21 ; 0.74)$.

\subsubsection{Linear regression part}

Pools from weaners and growers are expected to have lower CT values, in comparison with pools from breeding stock (-0.2 (95\% CI -0.34; -0.06), -0.13 (95\% CI -0.25; -0.01), respectively).

\subsection{PRRSV}

\subsubsection{Logistic regression part}

Presence of medium or high percentage of secondary respiratory infections in the farm is associated with higher probability of PRRSV seropositivity [OR 2.3 (95\% CI 1.16; 4.64)]. Mass vaccination until 3 days before farrowing and until 95 days of gestation is more likely to give positive CT values [OR $6.27(95 \%$ CI 3.17; 12.89)]. Also, vaccination of sows for PRRSV, weaners for PCV2 \& PRRSV and sows for PCV2 \& PRRSV (killed vaccine) is expected to increase PRRSV seropositivity [OR 7.28 (95\% CI 2.59; 23.36)]. Pool from weaners, growers and finishers are associated with PRRSV seropositivity [OR 12.03 (95\% CI 4.54; $35.53)$, OR 7.87 (95\% CI 2.96; 23.08), OR 3.91 (95\% CI 1.2; 13.39) respectively], compared to pools that come from breeding stock.

3.2.2 Linear regression part 
Nucleus breeding is associated with higher CT values, (0.15 (95\% CI 0.07; 0.24), if the other predictors in the model are held constant. Also, farms with "medium" / "adequate" biosecurity level are associated with lower CT values $(-0.08$ (95\% CI $-0.15 ;-0.01)$ if the other variables included in the model are held constant.

\section{DISCUSSION}

Control of both PRRSV and PCV2 relies in four different aspects: early diagnosis and monitoring, biosecurity level, herd management and immunization. Despite the different strategies that have been applied in order to minimize the PRRSV and PCV2 prevalence, both remain a huge problem in the worldwide swine industry. So, we designed a study in order to describe the prevalence of these two viruses among pig farms in Greece and associate the possible risk factors and PRRSV/PCV2 PCR-status in these herds. The survey provides useful information about the surveillance of PRRSV and PCV2 at national level, revealing data for the prevalence of the viruses and main risk factors for their monitoring.

Concerning the PRRSV circulation in pig farms, vaccination could be a key component to reduce the severity and frequency of the virus related problems and significantly contributes in the control of the infection. However, the efficacy of current PRRSV vaccines in the field is not known with precision and very few data are available regarding their usefulness for reducing PRRSV transmission. Based on the results of our study, vaccination schemes which could lead to an increased PRRSV circulation in the farms are the mass MLV PRRSV vaccination programs in breeding stock, especially during the last stages of gestation (until 3 days before farrowing) and the KV vaccination of sows during the middle of gestation. Regarding our observations about the mass MLV PRRSV vaccination, they can be explained by the safety concerns of MLV virus vaccines that have been reported in many studies. Many cases of vertical and horizontal transmission of the virus, due to MLV vaccines, have been demonstrated (Nielsen et al., 1997; Nielsen et al., 2001; Dewey, Wilson, Buck, \& Levenaar, 1999; Mengeling, Vorwald, Lager, Clouser, \& Wesley, 1999; Renukaradhya, Meng, Calvert, Roof, \& Lager, 2015). Specifically, there have been reports describing clinical cases in which despite the MLVs had achieved replication of the vaccine virus in vaccinated animals, subsequently the virus had been transmitted to naïve animals (Velasova et al., 2012). Additionally, the vaccine virus can cross the placental barrier, particularly if sows are vaccinated after the 90th day of their gestation and provoke transplacental infection in the fetuses followed by a possible subsequent death (Scortti, Prieto, Alvarez, Simarro, \& Castro, 2007; Pejsak, Podgorska, Truszczynski, Karbowiak, \& Stadejek, 2006). Our results about seropositivity in PRRSV MLV vaccinated farms may be also explained by the fact that PRRSV, as an RNA virus, is prone to mutation and its diversity continues to increase (Suarez et al., 1996; Drew, Lowings, \& Yapp, 1997; Frossard et al., 2008). The increasing genetic diversity may result in strains, which break through the efficacy of current PRRS vaccines and undermine PRRS control based on the use of vaccination only (Fraile et al., 2009). Previous studies reported that PRRSV infection with different strains leads to different virological and immunological outcomes and results in different degrees of homologous and heterologous protection (Diaz et al., 2012), as well as that different PRRSV strains differ in their susceptibility to antibody neutralization (Martínez-Lobo, Diez-Fuertes, Simarro, Castro, \& Prieto, 2011). Therefore, PRRSV MLVs may provide partial cross-protection against a PRRSV infection. Nevertheless, they could achieve a significant reduction in the frequency and severity of the virus outbreaks in commercial pig populations or even assist to eliminate the disease (Scortti et al., 2007; Papatsiros, 2012b; Savard et al., 2016; Piontkowski et al., 2016; Oh et al., 2014; Bitsouni et al., 2019).

Regarding the KV PRRSV vaccination our results are consistent with previous studies, which described their protective efficacy against the virus. Specifically, they reported that the KV vaccines may fail to reduce viremia, duration, titers of virus shedding and prevent reproductive losses (Zuckermann et al., 2007; Kim et al., 2011). However, in contrast to what our survey has described, a recent study suggested that the KVs could, effectively, cease the virus transmission or eliminate PRRSV from herds, resulting in drastic reduction infection prevalence, if it is adequately applied (Bitsouni et al., 2019). This contradiction to our results could be justified by the beneficial effects of PRRSV KV vaccines on speeding up recovery and potentially reducing infectivity of the pigs (Bassaganya-Riera et al., 2004; Kim et al., 2011). For this reason, they have been recommended for use as therapeutic vaccines for PRRSV treatment rather than for disease prevention, 
as they can decrease pathogen shedding in seropositive animals and improve the reproductive performance, e.g. increased farrowing rate, number of weaned pigs, health status of piglets born to vaccinated sows (Kim et al., 2011; Papatsiros et al., 2006; Zuckermann et al., 2007).

In addition, we observed that the vaccination of weaners against PCV2 and PRRSV (MLV vaccine) is expected to increase PRRSV circulation in the farm. These results are in contrast to previous studies reported that MLV PRRSV vaccination of weaners followed by a PCV2 vaccination had a beneficial effect in decreasing PRRS viremia and the pigs were protected against PRRS, but they showed increased PCV2 replication (Fraile et al., 2009; Niederwerder et al., 2015). This disagreement could be explained either from partial protection of applied commercial MLV PRRSV vaccines against field strains or wrong time of vaccination; near to time of infection, not providing enough time for efficient immunity to be raised against PRRSV infection. As mentioned above, the PRRS MLVs provide partial virological and clinical protection against heterologous PRRSV strains and given that the virus is highly prone to mutation most challenges in the field can be considered as heterologous. Consequently, the MLVs may be less effective against PRRS. However, at a herd level, the efficacy of the MLV vaccines should be evaluated in both virological and epidemiological terms. Specifically, the goals of a proper vaccination scheme against PRRSV are not only the animal's clinical protection, but also the ceasing of virus transmission in the pig farm. Therefore, the potential efficacy of MLV vaccines can be estimated through the evaluation of the biological parameters related to transmission and the determination of the virus reproduction rate (Pileri \& Mateu, 2016). Our results further indicate that a combined approach of surveillance for infection and PRRSV laboratory diagnosis is necessary in order to control or even eliminate the PRRSV from the farms. Generally it is very important in order to design a vaccination program against PRRSV to: a) investigate the serological profile, in order to determine the time of natural infection, b) to determine the field strain and compare the results of the sequencing analysis with the commercial MLV vaccines (Velasova et al., 2012).

PRRSV infects and compromises the function of pulmonary alveolar and intravascular macrophages resulting in a number of immunological outcomes that increase the animal's susceptibility to secondary infections caused by other pathogens (Pol, Van Leengoed, Stockhofe, Kok, \& Wensvoort, 1997; Solano, Segales, Collins, Molitor, \& Pijoan, 1997; Kim, Chung, \& Chae, 2003; Renukaradhya, Alekseev, Jung, Fang, \& Saif, 2010; Opriessnig et al., 2011; Gómez-Laguna, Salguero, Pallares, \& Carrasco, 2013; Thanawongnuwech et al., 2000; Thanawongnuwech, Thacker, Halbur, \& Thacker, 2004; Wills et al., 2000). Our results confirmed that the presence of medium or high percentage of secondary respiratory infections in the farm caused by other pathogens is associated with higher probability of PRRSV seropositivity. Additionally, we found that weaners, growers and finishers were more likely to show PRRSV seropositivity compared to breeding stock. Several studies whose findings are consistent with ours, described clinical cases of persistence of PRRSV infection in a breeding herd, which were caused by mixing susceptible with infected pigs in later finishing stages. Indeed after a PRRSV outbreak a subpopulation of pigs remain susceptible and can be subsequently be infected in different stages of production (Nodelijk, 2003). Therefore, where susceptible and infectious pigs are mixed, such as at weaning/growing/finishing house, various sub-populations of the herd may be infected.

Increased purchase of sows/gilts and semen without any previous serological examination alongside with poor biosecurity level were shown to be significant risk factors for PRRSV infection at herd level. These results are in agreement with previous studies, which described several cases of virus introduction through these routes. For example, Mortensen et al. (1992) associated the spread of PRRSV in a Danish herd to the purchase of infected sows and gilts without performing any previous serological examination or quarantine measures. Similar case reports of herd infections due to introduction of infected pigs were reported in Canada (Rosendal et al, 2016). The use of contaminated semen has, also, reported as an important transmission route of PRRSV into a farm. Specifically, Botner et al. (2000) demonstrated that the clinical outbreaks occurring in Danish PRRS- free breeding herds were caused by the import of contaminated semen. Additionally, several works reported clinical cases of PRRS infection in which trucks, trailers and other vehicles used for transporting pigs, feed or animal products were responsible for the virus spread. It was shown that the virus could be isolated from the ventral surface of transport vehicles, the truck wash floor, drivers' boots or from the surface 
of various types of containers (Dee, 2003).

Concerning the PCV2 circulation in pig farms, since commercial vaccines against PCV2 became commonly available in 2006, their use lead to control clinical signs and economical losses, characterized by improvement of performance parameters and a declining level of PCV2 prevalence and viremia in the field (Pejsak et al., 2010; Kristensen et al., 2011; Kristensen et al., 2018; Martelli et al., 2011; Martelli et al., 2016; Chae, 2012; Opriessnig et al., 2014a; Opriessnig et al., 2014b; Segalés, 2015; Tzika, 2015; Dvorak et al., 2016; Afghah et al., 2017; Tassis et al., 2017; Figueras-Gourgues et al., 2019). Nevertheless, we found several risk factors associated with PCV2 circulation in herds. Specifically breeding stock was more likely to be associated with PCV2 circulation (PCR positive CT values) compared to weaners and growers. Recent studies, whose findings are similar to ours, revealed that the spread and maintenance of PCV2 infection in farms was mainly due to gilts from the quarantine and rearing area and sows up to the second parity, which were viraemic (Eddicks et al., 2019). In addition, it has been reported that a one-year mass PCV2 vaccination was able to improve pigs performance parameters, but not to eradicate the virus infection and PCV2 became detectable again when vaccination was stopped (Feng, Blanco, Segales, \& Sibila, 2014). Another explanation of our results may be the absence of vaccination programs against PCV2 in breeding stock, which is applied in the majority of Greek farms, resulting in the maintenance of PCV2 circulation in sows. However, Lin, Ke, \& Chiou (2020) reported that natural exposure of PCV2 occurs in the growing to fattening period and viraemia can last until slaughter.

Moreover, we observed that farms located in Western Greece are less likely to be PCV2 seropositive compared to farms located in other regions of Greece (Southern/Northern/Eastern Greece). Recent surveys reported the role of the wide PCV2 inoculation in the determination of a differential fitness among genotypes, which may affect the virus epidemiology and evolution (Franzo et al., 2015; Franzo, Cortey, Segales, Hughes, \& Drigo, 2016). Therefore, our observation arises questions about the efficacy of vaccinations programs against PCV2 or the contact network between farms, which could play an important role due to introduction of animals from vaccinated farms in which different PCV2 strains may circulate. Based on our results, further studies are required in order to investigate the strains that are circulating in pig populations in Greece. Furthermore, our results showed that the vaccination of weaners for PCV2 is associated with small probability of PCV2 seropositivity, while breeding stock is more likely to be associated with positive CT values compared to weaners, indicating that PCV2 infection in a herd can be maintained thanks to the presence and the circulation of the virus within the breeding herd and thus shedding by gilts and young sows in farrowingunits.

Recently epidemiological studies focused on the development platforms as a tool, which allows the rapid visualization of PRRSV, including stakeholder information, for monitoring PRRSV infection at the national level (Torrents et al., 2019; Trevisan et al., 2019). Our results could be the start for the development of similar platforms for a national surveillance-monitoring program for PRRSV and PCV2 in Greece. Similar future molecular epidemiological studies could help to understand further and explore the extent of crossimmunity between field and vaccine strains, in order develop efficient monitoring strategies that might be able to diminish PRRSV and PCV2 impact on swine industry.

\section{ACKNOWLEDGEMENTS}

This work was supported by Zoetis Hellas S.A. through the Research Committee of the University of Thessaly, Greece (grant number: 6252, Scientific Responsible: Associate Professor V. Papatsiros).

\section{CONFLICT OF INTERESTS}

The authors declare no conflict of interest of any kind arising out this manuscript.

\section{ETHICAL APPROVAL}

The authors confirm that the ethical policies of the journal, as noted on the journal's author guidelines page, have been adhered to. No ethical approval was required as this report solely contains diagnostic samples taken in the field. 


\section{DATA AVAILABILITY STATEMENT}

The data that support the findings of this study are available from the corresponding author upon reasonable request.

\section{REFERENCES}

Afghah, Z., Webb, B., Meng, X. J., Ramamoorthy, S. (2017). Ten years of PCV2 vaccines and vaccination: Is eradication a possibility? Veterinary Microbiology, 206 :21-28. doi: 10.1016/j.vetmic.2016.10.002.

Alexopoulos, C., Kritas, S. K., Kyriakis, C. S., Tzika, E., Kyriakis, S. C. (2005). Sow performance in an endemically porcine reproductive and respiratory syndrome (PRRS)-infected farm after sow vaccination with an attenuated PRRS vaccine. Veterinary Microbiology,111 :151-157. doi: 10.1016/j.vetmic.2005.10.007.

Bassaganya-Riera, J., Thacker, B. J., Yu, S., Strait, E., Wannemuehler, M. J., Thacker, E. L. (2004). Impact of immunizations with porcine reproductive and respiratory syndrome virus on lymphoproliferative recall responses of CD8+ T cells. Viral Immunology,17 :25-37. doi: 10.1089/088282404322875430.

Baysinger, A. K., Dewey, C. E., Straw, B. E., Brumm, M. C., Schmitz, J., Doster, A., Kelling, C. (1997). Risk factors associated with endemic reproductive deficiencies caused by PRRSV infection. Journal of Swine Health and Production, 5 :179-87. doi.org/10.1186/s13028-018-0411-7.

Bitsouni, V., Lycett, S., Opriessnig, T., Doeschl-Wilson, A. (2019). Predicting vaccine effectiveness in livestock populations: A theoretical framework applied to PRRS virus infections in pigs. PloS one,14 (8):e0220738. doi.org/10.1371/journal.pone.0220738.

Bøtner, A., Strandbygaard, B., Sørensen, K.J., Oleksiewicz, M.B., Storgaard, T. (2000). Distinction between infections with European and American/vaccine type PRRS virus after vaccination with a modified-live PRRS virus vaccine. Veterinaty Research , 31 :72-72. doi: 10.1051/vetres:2000009.

Cano, J. P., Dee, S. A., Murtaugh, M. P., Pijoan, C. (2007). Impact of a modified-live porcine reproductive and respiratory syndrome virus vaccine intervention on a population of pigs infected with a heterologous isolate. Vaccine 25 :4382-4391. doi:10.1016/j.vaccine.2007.03.031.

Dee, S. A. (2003). Approaches to prevention, control, and eradication. PRRS Compendium. In: Zimmerman J, Yoon K-J, editors. The PRRS Compendium . (2nd ed.) Des Moines, Iowa: National Pork Board.

Dewey, C. E., Wilson, S., Buck, P., Leyenaar, J. K. (1999). The reproductive performance of sows after PRRS vaccination depends on stage of gestation. Preventive Veterinary Medicine,11 :233-241. doi: 10.1016/s01675877(99)00025-2.

Díaz, I., Gimeno, M., Darwich, L., Navarro, N., Kuzemtseva, L., López, S., Galindo, I., Segalés, J., Martín, M., Pujols, J., Mateu, E. (2012). Characterization of homologous and heterologous adaptive immune responses in porcine reproductive and respiratory syndrome virus infection. Veterinary Research, 43 :30-10. doi: 10.1186/1297-9716-43-30.

Drew, T. W., Lowings, J. P., Yapp, F. (1997). Variation in open reading frames 3, 4 and 7 among porcine reproductive and respiratory syndrome virus isolates in the UK. Veterinary Microbiology,55 :209-221. doi: 10.1016/s0378-1135(96)01328-4.

Drolet, R., Larochelle, R., Morin, M., Delisle, B., Magar, R. (2003). Detection rates of porcine reproductive and respiratory syndrome virus, porcine circovirus type 2 , and swine influenza virus in porcine proliferative and necrotizing pneumonia. Veterinary Pathology,40 :143-148. doi: 10.1354/vp.40-2-143.

Duinhof, T. F., Van Schaik, G., Van Esch, E. J., Wellenberg, G. J. (2011). Detection of PRRSV circulation in herds without clinical signs of PRRS: comparison of five age groups to assess the preferred age group and sample size. Veterinary Microbiology, 150 :180-184. doi: 10.1016/j.vetmic.2011.01.001. 
Dvorak, C. M., Yang, Y., Haley, C., Sharma, N., Murtaugh, M. P. (2016). National reduction in porcine circovirus type 2 prevalence following introduction of vaccination. Veterinary Microbiology,189:86-90. doi: 10.1016/j.vetmic.2016.05.002.

EC (European Council). (2003). Directive 2003/65/EC of the European Parliament and of the Council of 22 July 2003 amending Council Directive 86/609/EEC on the approximation of laws, regulations and administrative provisions of the member states regarding the protection of animals used for experimental and other scientific purposes. O J EEC; L230 :32-33.

Eddicks, M., Beuter, B., Stuhldreier, R., Nolte, T., Reese, S., Sutter, G., Ritzmann, M., Fux, R. (2019). Crosssectional study on viraemia and shedding of porcine circovirus type 2 in a subclinically infected multiplier sow herd. Veterinary Record, 184 :189. doi: 10.1136/vr.105069.

EMEA (European Medicines Agency). (2001). Committee for veterinary medicinal products: note for guidance field trials with veterinary vaccines. EMEA/CVMP/852/99-FINAL.

Evans, C. M., Medley, G. F., Green, L. E. (2008). Porcine reproductive and respiratory syndrome virus (PRRSV) in GB pig herds: farm characteristics associated with heterogeneity in seroprevalence.BMC Veterinary Research, 4 :48-10. doi.org/10.1186/1746-6148-4-48.

Fan, P., Wei, Y., Guo, L., Wu, H., Huang, L., Liu, J., Liu, C. (2013). Synergistic effects of sequential infection with highly pathogenic porcine reproductive and respiratory syndrome virus and porcine circovirus type 2 . Virology Journal, 10 :265. doi.org/10.1186/1743-422X-10-265.

FASS (Federation of Animal Science Societies). (2010). Guide for the care and use of agricultural animals in research and teaching. Guide for the Care and Use of Agricultural Animals in Research and Teaching. 3rd ed. Champagne, IL: FASS.

Feng, H., Blanco, G., Segalés, J., Sibila, M. (2014). Can Porcine circovirus type 2 (PCV2) infection be eradicated by mass vaccination?. Veterinary Microbiology, 172 (1-2): 92-99. doi: 10.1016/j.vetmic.2014.05.003.

Figueras-Gourgues, S., Fraile, L., Segalés, J., Hernández-Caravaca, I., López-Úbeda, R., Garcia-Vazquez, F., Duran, O., Grosse-Liesner, B. (2019). Effect of Porcine circovirus 2 (PCV-2) maternally derived antibodies on performance and PCV-2 viremia in vaccinated piglets under field conditions. Porcine Health Management, 5 :21. doi.org/10.1186/s40813-019-0128-7.

Fraile, L., Calsamiglia, M., Mateu, E., Espinal, A., Cuxart, A., Seminati, C., Martín, M., Domingo, M., Segalés, J. (2009). Prevalence of infection with porcine circovirus-2 (PCV-2) and porcine reproductive and respiratory syndrome virus (PRRSV) in an integrated swine production system experiencing postweaning multisystemic wasting syndrome. Canadian Journal of Veterinary Research, 73 (4):308-312.

Franzo, G., Tucciarone, C. M., Dotto G., Gigli, A., Ceglie, L., Drigo M. (2015). International trades, local spread and viral evolution: The case of porcine circovirus type 2 (PCV2) strains heterogeneity in Italy.Infection, Genetics and Evolution, 32 . doi: 10.1016/j.meegid.2015.04.004.

Franzo, G., Cortey, M., Segales, J., Hughes, J., Drigo, M. (2016). Phylodynamic analysis of porcine circovirus type 2 reveals global waves of emerging genotypes and the circulation of recombinant forms.Molecular Phylogenetics and Evolution, 100 :269-280. doi: 10.1016/j.ympev.2016.04.028.

Frossard, J. P., Westcott, D. G., Naidu, B., Drew, T. W., Williamson, S., Woodger, N. G. A. (2008). Porcine reproductive and respiratory syndrome virus: diversity of recent British isolates. Pig Journal, 61 :36-41.

Gillespie, J., Opriessnig, T., Meng, X. J., Pelzer, K., Buechner-Maxwell, V. (2009). Porcine circovirus type 2 and porcine circovirus-associated disease. Journal of Veterinary Internal Medicine, 23 (6):1151-1163. doi: 10.1111/j.1939-1676.2009.0389.x.

Gómez-Laguna, J., Salguero, F. J., Pallares, F. J., Carrasco, L. (2013). Immunopathogenesis of porcine 
reproductive and respiratory syndrome in the respiratory tract of pigs. The Veterinary Journal,195:148155. doi.org/10.1016/j.tvj1.2012.11.012.

Harms, P. A., Sorden, S. D., Halbur, P. G., Bolin, S. R., Lager, K. M., Morozov, I., Paul, P. S. (2001). Experimental reproduction of severe disease in $\mathrm{cd} / \mathrm{cd}$ pigs concurrently infected with type 2 porcine circovirus and porcine reproductive and respiratory syndrome virus. Veterinary Pathology, 38 :528-539. doi.org/10.1354/vp.38-5-528.

Incerti, D. (2015). An Assessment of Long-term Healthcare Expenditure Risk using a Dynamic Bayesian Model.

Kim, H., Kim, H. K., Jung, J. H., Choi, Y. J., Kim, J., Um, C. G., Hyun, S. B., Shin, S., Lee, B., Jang, G., Kang, B. K., Moon, H. J., Song, D. S. (2011). The assessment of efficacy of porcine reproductive respiratory syndrome virus inactivated vaccine based on the viral quantity and inactivation methods. Virology Journal, 8 (1):323. doi: 10.1186/1743-422X-8-323.

Kim, J., Chung, H. K., Chae, C. (2003). Association of porcine circovirus 2 with porcine respiratory disease complex. The Veterinary Journal, 166 :251-256. doi: 10.1016/s1090-0233(02)00257-5.

Kristensen C. S., Kvisgaard L. K., Pawlowski, M., Carlsen, S. H., Hjulsager, C. K., Heegaard, P. M. H., Bøtner, A., Stadejek, T., Haugegaard, S., Larsen, L. E. (2018). Efficacy and safety of simultaneous vaccination with two modified live virus vaccines against porcine reproductive and respiratory syndrome virus types 1 and 2 in pigs. Vaccine, 36 (2):227-236. doi: 10.1016/j.vaccine.2017.11.059.

Kristensen, C. S., Baadsgaard, N. P., Toft, N. (2011). A meta-analysis comparing the effect of PCV2 vaccines on average daily weight gain and mortality rate in pigs from weaning to slaughter. Preventive Veterinary Medicine, 98 :250-258. doi: 10.1016/j.prevetmed.2010.11.015.

Lin, C. N., Ke, N. J., Chiou, M. T. (2020). Cross-Sectional Study on the Sero- and Viral Dynamics of Porcine Circovirus Type 2 in the Field. Vaccines, 8 :339. doi.org/10.3390/vaccines8020339.

Martelli, P., Ferrari, L., Morganti, M., De Angelis, E., Bonilauri, P., Guazzetti, S., Caleffi, A., Borghetti, P. (2011). One dose of a porcine circovirus 2 subunit vaccine induces humoral and cell-mediated immunity and protects against porcine circovirus-associated disease under field conditions. Veterinary Microbiology, 149 :339-351. doi: 10.1016/j.vetmic.2010.12.008.

Martelli, P., Saleri, R., Ferrarini, G., De Angelis, E., Cavalli, V., Benetti, M., Ferrari, L., Canelli, E., Bonilauri, P., Arioli, E. (2016). Impact of maternally derived immunity on piglets' immune response and protection against porcine circovirus type 2 (PCV2) after vaccination against PCV2 at different age. BMC Veterinary Research,12 :77. doi: 10.1186/s12917-016-0700-1.

Martínez-Lobo, F.J., Díez-Fuertes, F., Simarro, I., Castro, J.M., Prieto, C. (2011). Porcine Reproductive and Respiratory Syndrome Virus isolates differ in their susceptibility to neutralization. Vaccine, 29 :6928-6940. doi: 10.1016/j.vaccine.2011.07.076.

Mateu, E., Diaz, I. (2008). The challenge of PRRS immunology. The Veterinary Journal, 177 (3):345-351. doi: 10.1016/j.tvjl.2007.05.022.

Mengeling, W. L., Vorwald, A. C., Lager, K. M., Clouser, D. F., Wesley, R. D. (1999). Identification and clinical assessment of suspected vaccine-related field strains of porcine reproductive and respiratory syndrome virus. American Journal of Veterinary Research,60 :334-340.

Mortensen, S., Stryhn, H., Sogaard, R., Boklund, A., Stark, K. D., Christensen, J., Willeberg, P. (2002). Risk factors for infection of sow herds with porcine reproductive and respiratory syndrome (PRRS) virus.Preventive Veterinary Medicine, 53 (1-2):83-101. doi: 10.1016/s0167-5877(01)00260-4.

Nan, Y., Wu, C., Gu, G., Sun, W., Zhang, Y. J., Zhou, E. M. (2017). Improved vaccine against PRRSV: current progress and future perspective.Frontiers in Microbiology, 8 :1635. doi: 10.3389/fmicb.2017.01635. 
Niederwerder, M. C., Bawa, B., Serão, N. V., Trible, B. R., Kerrigan, M. A., Lunney, J. K., Dekkers, J. C., Rowland, R. R. (2015). Vaccination with a Porcine Reproductive and Respiratory Syndrome (PRRS) Modified Live Virus Vaccine Followed by Challenge with PRRS Virus and Porcine Circovirus Type 2 (PCV2) Protects against PRRS but Enhances PCV2 Replication and Pathogenesis Compared to Results for Nonvaccinated Cochallenged Controls. CVI 22 (12):1244-1254. doi: 10.1128/CVI.00434-15.

Nielsen, H. S., Oleksiewicz, M. B., Forsberg, R., Stadejek, T., B tner, A., Storgaard, T. (2001). Reversion of a live porcine reproductive and respiratory syndrome virus vaccine investigated by parallel mutations. Journal of General Virology, 82 :1263-1272. doi: 10.1099/0022-1317-82-6-1263.

Nielsen, T. L., Nielsen, J., Have, P., Baekbo, P., Hoff-Jorgensen, R., B tner, A. (1997). Examination of virus shedding in semen from vaccinated and from previously infected boars after experimental challenge with porcine reproductive and respiratory syndrome virus. Veterinary Microbiology, 5 :101-112. doi.org/10.1016/S0378-1135(96)01272-2.

Nieuwenhuis, N., Duinhof, T. F., van Nes, A. (2012). Economic analysis of outbreaks of porcine reproductive and respiratory syndrome virus in nine sow herds. Veterinary Record, 170 :225. doi: 10.1136/vr.100101.

Nodelijk, G., Nielen, M., De Jong, M.C.M., Verheijden, J.H.M. (2003). A review of porcine reproductive and respiratory syndrome virus in Dutch breeding herds: population dynamics and clinical relevance.Preventive Veterinary Medicine, 60 :37-52. doi: 10.1016/S0167-5877(03)00081-3.

OECD (Organization for Economic Co-operation and Development). (2000). Guidance Document on the Recognition, Assessment, and Use of Clinical Signs as Humane Endpoints for Experimental Animals Used in Safety Evaluation. (ENV/JM/MONO(2000)7). Paris: OECD. Retrieved from http.7/www.occd.org/ehs/test/mono19.pdf .

Oh, Y., Seo, H. W., Park, C., Chae, C. (2014). Comparison of sow and/or piglet vaccination of 3 commercial porcine circovirus type 2 (PCV2) single-dose vaccines on pigs under experimental PCV2 challenge. Veterinary Microbiology, 172 :371-380. doi: 10.1016/j.vetmic.2014.05.028.

Opriessnig, T., Gimenez-Lirola, L. G., Halbur, P. G. (2011). Polymicrobial respiratory disease in pigs. Animal Health Research Reviews, 12 :133-148. doi: 10.1017/S1466252311000120.

Opriessnig, T., Gerber, P. F. Xiao, C. T., Halbur, P. G., Matzinger, S. R., Meng, X. J. (2014b). Commercial PCV2a-based vaccines are effective in protecting naturally PCV2b-infected finisher pigs against experimental challenge with a 2012 mutant PCV2. Vaccine,32 :4342-4348. doi: 10.1016/j.vaccine.2014.06.004.

Opriessnig, T., Gerber, P. F., Xiao, C. T., Mogler, M., Halbur, P. G. (2014a). A commercial vaccine based on $\mathrm{PCV} 2 \mathrm{a}$ and an experimental vaccine based on a variant $\mathrm{mPCV} 2 \mathrm{~b}$ are both effective in protecting pigs against challenge with a 2013 US variant mPCV2b strain. Vaccine,32 :230-237. doi: 10.1016/j.vaccine.2013.11.010.

Opriessnig, T., Madson, D. M., Prickett, J. R., Kuhar, D., Lunney, J. K., Elsener, J., Halbur, P. G. (2008). Effect of porcine circovirus type 2 (PCV2) vaccination on porcine reproductive and respiratory syndrome virus (PRRSV) and pcv2 coinfection. Veterinary Microbiology,131 :103-114. doi: 10.1016/j.vetmic.2008.03.002.

Ouyang, T., Zhang, X., Liu, X., Ren, L. (2019). Co-Infection of Swine with Porcine Circovirus Type 2 and Other Swine Viruses. Viruses,11 (2):185. doi: 10.3390/v11020185.

Papatsiros, V. G. (2012a). Porcine Herd Health Management Practices for the Control of PRRSV Infection. In: Perez-Marin CC (ed) A Bird's-Eye View of Veterinary Medicine. In-Tech, Croatia.

Papatsiros, V. G. (2012b) Porcine Respiratory and Reproductive Syndrome Virus vaccinology: a review for commercial vaccines. American Journal of Animal and Veterinary Sciences, 7 (4):149-158. doi: 10.3844/ajavssp.2012.149.158. 
Papatsiros, V. G., Alexopoulos, C., Kritas, S. K., Koptopoulos, G., Nauwynck, H. J., Pensaert, M. B., Kyriakis, S. C. (2006). Long-term administration of a commercial porcine reproductive and respiratory syndrome virus (PRRSV)-inactivated vaccine in PRRSV-endemically infected sows. Journal of Veterinary Medicine B. Infectious Diseases and Veterinary Public Health, 53 : 266-272. doi: 10.1111/j.1439-0450.2006.00965.x.

Pearson, K. (1900). X. On the criterion that a given system of deviations from the probable in the case of a correlated system of variables is such that it can be reasonably supposed to have arisen from random sampling. The London, Edinburgh, and Dublin Philosophical Magazine and Journal of Science, 50 (302):157-175. doi: $10.2307 / 1402731$

Pejsak, Z., Podgórska, K., Truszczyński, M., Karbowiak, P., Stadejek, T. (2010). Efficacy of different protocols of vaccination against porcine circovirus type 2 (PCV2) in a farm affected by postweaning multisystemic wasting syndrome (PMWS). Comparative Immunology, Microbiology $\& 3$ Infectious Diseases, 33 (6):e1-5. doi: 10.1016/j.cimid.2009.09.006.

Pileri, E., \& Mateu, E. (2016). Review on the transmission porcine reproductive and respiratory syndrome virus between pigs and farms and impact on vaccination. Veterinary Research, 47 :108. doi: 10.1186/s13567016-0391-4.

Piontkowski, M. D., Kroll, J., Orveillon, F. X., Kraft, C., Coll, T. (2016). Safety and efficacy of a novel European vaccine for porcine reproductive and respiratory virus in bred gilts. The Canadian Journal of Veterinary Research, 80 (4):269-280.

Pol, J. M., Van Leengoed, L. A., Stockhofe, N., Kok, G., Wensvoort, G. (1997). Dual infections of PRRSV/influenza or PRRSV/Actinobacillus pleuropneumoniae in the respiratory tract. Veterinary Microbiology, 55 :259-264. doi.org/10.1016/S0378-1135(96)01323-5.

Core Team R. (2019). R: A language and environment for statistical computing. R Foundation for Statistical Computing, Vienna, Austria. Retrieved from https://www.R-project.org/.

Renukaradhya, G. J., Alekseev, K., Jung, K., Fang, Y., Saif, L. J. (2010). Porcine reproductive and respiratory syndrome virus-induced immunosuppression exacerbates the inflammatory response to porcine respiratory coronavirus in pigs. Viral Immunology,23 :457-466. doi: 10.1089/vim.2010.0051.

Renukaradhya, G. J., Meng, X. J., Calvert, J. G., Roof, M., Lager, K. M. (2015). Inactivated and subunit vaccines against porcine reproductive and respiratory syndrome: Current status and future direction. Vaccine, 33 (27):3065-3072. doi: 10.1016/j.vaccine.2015.04.102.

Rose, N., Blanchard, P., Cariolet, R., Grasland, B., Amennay, N., Oger, A., Durandz, B., Balaschy, M., Jestin, A., Madec, F. (2007). Vaccination of porcine circovirus type 2 (PCV2)-infected sows against porcine parvovirus (PPV) and erysipelas: effect on post-weaning multisystemic wasting syndrome (PMWS) and on PCV2 genome load in the offspring. Journal of Comparative Pathology, 136 :133-144. doi: 10.1016/j.jcpa.2007.01.006.

Rosendal, T., Dewey, C., Friendship, R., Wootton, S., Young, B., Poljak, Z. (2016). Association between PRRSV ORF5 genetic distance and differences in space, time, ownership and animal sources among commercial pig herds. Transboundary and Emerging Diseases,63 :e185-93.doi: 10.1111/tbed.12253.

Savard, C., Alvarez, F., Provost, C., Chorfi, Y., D’Allaire, S., Benoit-Biancamano, M. O., Gagnon, C. A. (2016). Efficacy of Fostera PRRS modified live virus vaccine against a Canadian heterologous virulent field strain of porcine reproductive and respiratory syndrome virus. Canadian Journal of Veterinary Research, $\mathbf{8 0}$ (1):1-11.

Scortti, M., Prieto, C., Alvarez, E., Simarro, I., Castro, J. (2007). Failure of an inactivated vaccine against porcine reproductive and respiratory syndrome to protect gilts against a heterologous challenge with PRRSV. Veterinary Record, 161 (24):809-813. 
Segalés, J. (2015). Best practice and future challenges for vaccination against porcine circovirus type 2 . Expert Review of Vaccines,14 :473-487. doi: 10.1586/14760584.2015.983084.

Skampardonis, V., Sotiraki, S., Kostoulas, P., Leontides, L. (2012). Factors associated with the occurrence and level of Isospora suisoocyst excretion in nursing piglets of Greek farrow-to-finish herds. BMC Veterinary Research, 8 (1):228. doi.org/10.1186/1746-6148-8-228.

Solano, G. I., Segalés, J., Collins, J. E., Molitor, T. W., Pijoan, C. (1997). Porcine reproductive and respiratory syndrome virus (PRRSv) interaction with Haemophilus parasuis . Veterinary Microbiology, $\mathbf{5 5}: 247-257$. doi: 10.1016/s0378-1135(96)01325-9.

Suarez, P., Zardoya, R., Martin, M. J., Prieto, C., Dopazo, J., Solana, A., Castro, J. M. (1996). Phylogenetic relationships of European strains of porcine reproductive and respiratory syndrome virus (PRRSV) inferred from DNA sequences of putative ORF-5 and ORF-7 genes. Virus Research, 42 (1-2):159-165. doi.org/10.1016/0168-1702(95)01305-9.

Sun, J., Huang, L., Wei, Y., Wang, Y., Chen, D., Du, W., Wu, H., Liu, C. (2015). Prevalence of emerging porcine parvoviruses and their co-infections with porcine circovirus type 2 in China. Archives of Virology, 160 :1339-1344. doi.org/10.1007/s00705-015-2373-7.

Tassis, P. D., Tsakmakidis, I., Papatsiros, V. G., Koulialis, D., Nell, T., Brellou, G., Tzika, E. D. (2017). A randomized controlled study on the efficacy of a novel combination vaccine against enzootic pneumonia (Mycoplasma hyopneumoniae) and porcine Circovirus type 2 (PCV2) in the presence of strong maternally derived PCV2 immunity in pigs.BMC Veterinary Research, 13 (1):91. doi: 10.1186/s12917-017-1014-7.

Thanawongnuwech, R., Brown, G. B., Halbur, P. G., Roth, J. A., Royer, R. L., Thacker, B. J. (2000). Pathogenesis of porcine reproductive and respiratory syndrome virus-induced increase in susceptibility toStreptococcus suis infection. Veterinary Pathology,37 :143-152. doi: 10.1354/vp.37-2-143.

Thanawongnuwech, R., Thacker, B., Halbur, P., Thacker, E. L. (2004). Increased production of proinflammatory cytokines following infection with porcine reproductive and respiratory syndrome virus and Mycoplasma hyopneumoniae . Clinical and Diagnostic Laboratory Immunology, 11 :901-908. doi: 10.1128/CDLI.11.5.901908.2004.

Torrents, D., Miranda, J., Pedrazuela, R., Gauger, P. C., Ramirez, A., Linhares D. C. L. (2019). Implementation of PRRSV status classification system in swine breeding herds from a large integrated group in Spain.Porcine Health Management, 5 :26. doi.org/10.1186/s40813-019-0134-9.

Trevisan, G., Linhares, L. C. M., Crim, B., Dubey, P., Schwartz, K. J., Burrough, E. R., Main, R. G., Sundberg, P., Thurn, M., Lages, P. T. F., Corzo, C. A., Torrison, J., Henningson, J., Herrman, E., Hanzlicek, G. A., Raghavan, R., Marthaler, D., Greseth, J., Clement, T., Christopher-Hennings, J., Linhares, D. C. L. (2019). Macroepidemiological aspects of porcine reproductive and respiratory syndrome virus detection by major United States veterinary diagnostic laboratories over time, age group, and specimen.PLoS One, 14 (10):e0223544. doi: 10.1371/journal.pone.0223544.

Tzika, E., Tassis, P., Koulialis, D., Papatsiros, V., Nell, T., Brellou, G., Tsakmakidis, I. (2015). Field efficacy study of a novel ready-to-use vaccine against mycoplasma hyopneumoniae and porcine circovirus type 2 in a Greek farm. Porcine Health Management, 1 :15. doi: 10.1186/s40813-015-0006-x.

Velasova, M., Alarcon, P., Williamson, S., Wieland, B. (2012). Risk factors for porcine reproductive and respiratory syndrome virus infection and resulting challenges for effective disease surveillance.BMC Veterinary Research, 8 :184. doi.org/10.1186/1746-6148-8-184.

Weigel, R. M., Firkins, L. D., Scherba, G. (2000). Prevalence and risk factors for infection with Porcine Reproductive and Respiratory Syndrome Virus (PRRSV) in swine herds in Illinois (USA). Veterinary Research, 31 (1):87-88. doi.org/10.1051/vetres:2000069. 
Wills, R. W., Gray, J. T., Fedorka-Cray, P. J., Yoon, K. J., Ladely, S., Zimmerman, J. J. (2000). Synergism between porcine reproductive and respiratory syndrome virus (PRRSV) and Salmonella choleraesuis in swine. Veterinary Microbiology, 71 :177-192.doi: 10.1016/S0378-1135(99)00175-3.

Xie, H., McHugo, G., Sengupta, A., Clark, R., Drake, R. (2004). A method for analyzing longitudinal outcomes with many zeros. Mental Health Services Research, 6 (4):239-246. doi: 10.1023/b:mhsr.0000044749.39484.1b.

Zuckermann, F. A., Garcia, E. A., Luque, I. D., Christopher-Hennings, J., Doster, A., Brito, M., Osorio, F. (2007). Assessment of the efficacy of commercial porcine reproductive and respiratory syndrome virus (PRRSV) vaccines based on measurement of serologic response, frequency of gamma-IFN-producing cells and virological parameters of protection upon challenge. Veterinary Microbiology, 123 :69-85. doi: 10.1016/j.vetmic.2007.02.009.

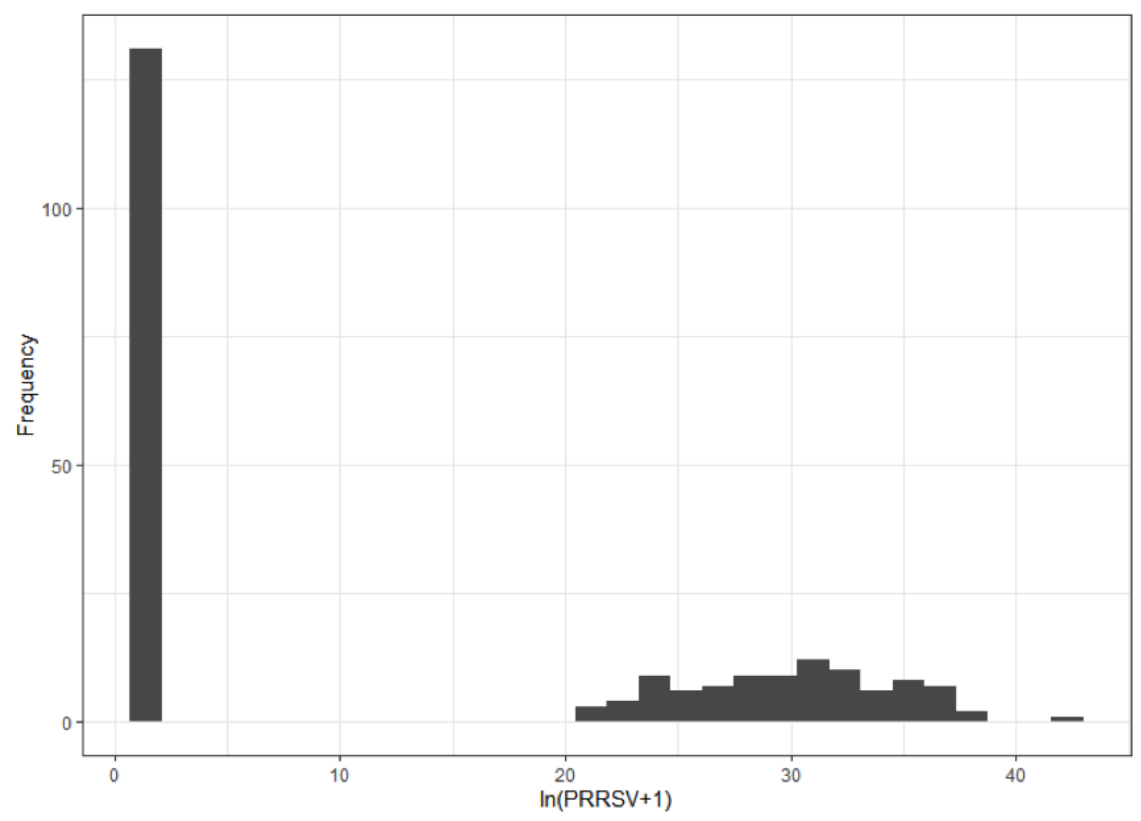

\section{Hosted file}

Tables.pdf available at https://authorea.com/users/376081/articles/493181-risk-factors-andprevalence-of-porcine-circovirus-2-and-porcine-reproductive-and-respiratory-virus-in-59greek-pig-farms 


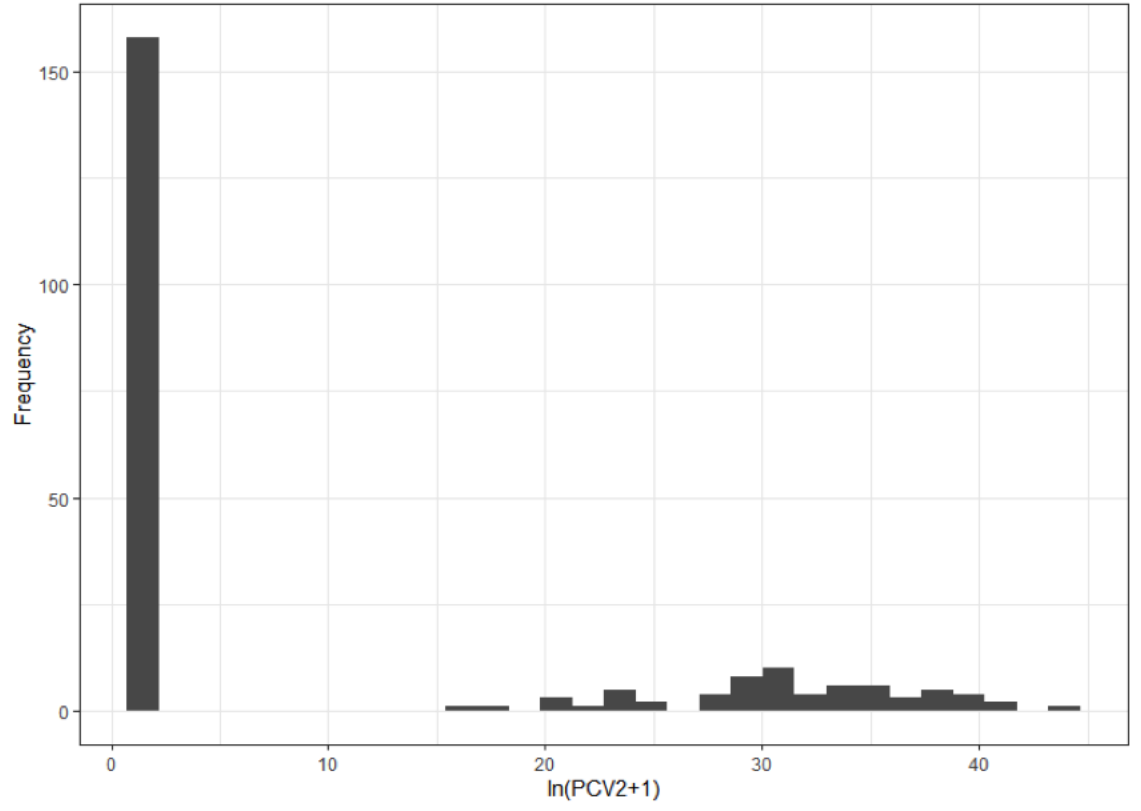

